\title{
Comment on "Resolution of the Einstein-Podolsky-Rosen and Bell Paradoxes"
}

\author{
Alan Macdonald \\ Department of Mathematics \\ Luther College, Decorah, IA 52101, U.S.A. \\ macdonal@luther.edu
}

Phys. Rev. Lett. 49, 1215 (1982).

(Slightly modified.)

PACS: 03.65.Bz

In a recent letter, ${ }^{1}$ Pitowsky has given a model of electron spin in which "Every electron at each given moment has a definite spin in all directions", but which, he claims, does not imply Bell's inequality. A non-Kolmogorov probability theory in the model prevents the usual proofs of Bell's inequality from going through. I give here a very simple proof of a Bell-type inequality from the quoted statement. The inequality shows that the statement is inconsistent with quantum mechanics.

Consider $N$ pairs of electrons in the singlet state. One member of each pair moves to the left and the other to the right. Let $N\left(A^{+}: C^{+}\right)$be the number of pairs in which the left member has spin up in the $A$ direction and the right member has spin up in the $C$ direction. Let $N\left(A^{+} C^{-}\right.$:) be the number in which the left member has spin up in the $A$ direction and spin down in the $C$ direction. According to the quoted statement, these are meaningful quantities. Then

$$
\begin{aligned}
N\left(A^{+}: C^{+}\right) & =N\left(A^{+} C^{-}:\right)=N\left(A^{+} B^{-} C^{-}:\right)+N\left(A^{+} B^{+} C^{-}:\right) \\
& \leq N\left(A^{+} B^{-}:\right)+N\left(B^{+} C^{-}:\right)=N\left(A^{+}: B^{+}\right)+N\left(B^{+}: C^{+}\right) .
\end{aligned}
$$

Quantum mechanics predicts that if $N\left(A^{+}: C^{+}\right)$is measured, then

$$
N\left(A^{+}: C^{+}\right) / N \approx \frac{1}{2} \sin ^{2} \frac{\theta_{A C}}{2},
$$

where $\theta_{A C}$ is the angle between $A$ and $C$. According to the quoted statement $N\left(A^{+}: C^{+}\right)$exists independently of whether it is measured or not and so the approximation holds whether it is measured or not. The above inequality is inconsistent with the approximation for $\theta_{A B}=\theta_{B C}=60^{\circ}$ and $\theta_{A C}=120^{\circ}$

${ }^{1}$ I. Pitowsky, Phys. Rev. Lett. 48, 1299 (1982). 